\title{
Designing a preliminary adaptive study to inform a biomarker trial in Psoriasis
}

\author{
Andrew T Prevost ${ }^{1 *}$, Jack Bowden ${ }^{2}$ \\ From Clinical Trials Methodology Conference 2011 \\ Bristol, UK. 4-5 October 2011
}

\section{Background}

Biomarkers play different roles in trials, being accordingly classified into 'prognostic', 'predictive', 'surrogate', or combinations thereof. Knowledge of a biomarker's role enables focused testing in late Phase trials through a better choice from available designs (e.g. 'stratified', 'strategy', and 'enrichment'). Preliminary studies can inform a biomarker's role and the relative value of multiple biomarkers.

\section{Motivation}

Here we consider the design of a preliminary study of potentially predictive biomarkers in patients treated for Psoriasis. A clinical researcher came for methodological advice, bringing a related published study with exciting results but of highly dubious quality. The objective was to provide a design with better properties (less bias, high power, low cost), allowing multiple biomarkers and their combination to be assessed to inform any subsequent trial.

\section{Methods}

Prior preferences, agreed by the researcher, were for a prospective design, control groups, and power-based sample size calculation. A formal 10-minute presentation to the full team was required to explain pros and cons of an adaptive element (two recruitment stages) to the design. Power was assessed through simulation in Rsoftware using Fisher's method, involving the product of stage p-values.

\section{Results}

Effect size was defined in terms of the correlation between treatment response over time and a biomarker.

\footnotetext{
* Correspondence: toby.prevost@kcl.ac.uk

'Department of Primary Care and Public Health Sciences, King's College London, SE1 3QD, UK

Full list of author information is available at the end of the article
}

Under a non-adaptive design, an R-squared of $20 \%$ could be detected with $90 \%$ power, $5 \%$ significance level, with 49 patients, with all 17 expensive biomarkers measured. The adaptive design offered an interesting alternative, employing $\mathrm{p}>0.3$ to discontinue with biomarkers quarter-way through recruitment, requiring $24+72=96$ patients. This offers more patients to develop a combination from an enriched biomarker set guaranteed to include the best five performers from stage one. The proportion of biomarkers expected to discontinue, conditional on underlying effect size, was considered graphically.

\section{Conclusions}

Incorporating methodological improvements into study designs requires understanding of methodology and collaborators. The cost-efficient two-stage design is an improvement on the related published study, and we outline further analysis-stage developments: reducing bias in estimates and providing valid confidence intervals and error rates $[1,2]$. The study proposal is currently going through ethics.

\section{Author details}

'Department of Primary Care and Public Health Sciences, King's College London, SE1 3QD, UK. ' Medical Research Council Biostatistics Unit, Institute of Public Health, Cambridge, CB2 OSR, UK.

Published: 13 December 2011

\section{References}

1. Bowden J, Glimm E: Unbiased estimation of selected treatment means in two-stage trials. Biom J 2008, 50:515-527.

2. Posch M, Koenig F, Branson M, Brannath W, Dunger-Baldauf $C$, Bauer P: Testing and estimation in flexible group sequential designs with adaptive treatment selection. Stat Med 2005, 24:3697-3714.

doi:10.1186/1745-6215-12-S1-A17

Cite this article as: Prevost and Bowden: Designing a preliminary adaptive study to inform a biomarker trial in Psoriasis. Trials 201112 (Suppl 1):A17.

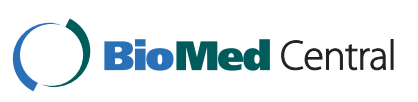

() 2011 Prevost and Bowden; licensee BioMed Central Ltd. This is an open access article distributed under the terms of the Creative Commons Attribution License (http://creativecommons.org/licenses/by/2.0), which permits unrestricted use, distribution, and reproduction in any medium, provided the original work is properly cited. 